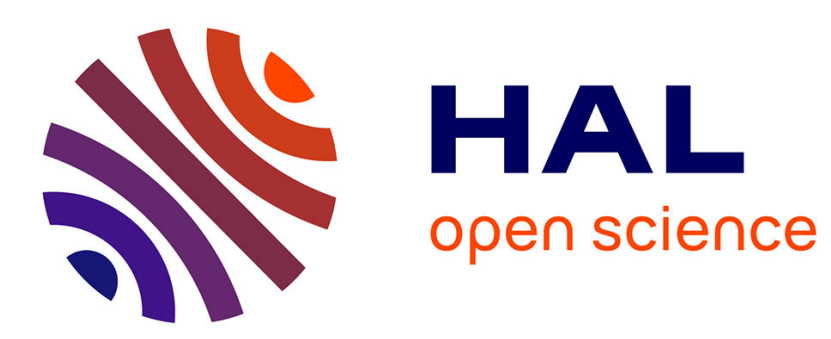

\title{
One-dimensional barcode reading: an information theoretic approach
}

\author{
Karim Houni, Wadih Sawaya, Yves Delignon
}

\section{To cite this version:}

Karim Houni, Wadih Sawaya, Yves Delignon. One-dimensional barcode reading: an information theoretic approach. Applied optics, 2008, Vol. 47, Issue 8, pp. (2008) (8), pp.1025-1036. 10.1364/AO.47.001025 . hal-00805824

\section{HAL Id: hal-00805824 https://hal.science/hal-00805824}

Submitted on 29 Mar 2013

HAL is a multi-disciplinary open access archive for the deposit and dissemination of scientific research documents, whether they are published or not. The documents may come from teaching and research institutions in France or abroad, or from public or private research centers.
L'archive ouverte pluridisciplinaire HAL, est destinée au dépôt et à la diffusion de documents scientifiques de niveau recherche, publiés ou non, émanant des établissements d'enseignement et de recherche français ou étrangers, des laboratoires publics ou privés. 


\title{
One-dimensional barcode reading: an information theoretic approach
}

\author{
Karim Houni, ${ }^{1, \star}$ Wadih Sawaya, ${ }^{1}$ and Yves Delignon ${ }^{2}$ \\ ${ }^{1}$ Telecom Lille 1, rue G. Marconi, 59650 Villeneuve d'Ascq, France \\ ${ }^{2}$ Groupe des Écoles des Télécommunications, Institut National des Télécommunications, Telecom Lille 1, rue G. Marconi, \\ 59650 Villeneuve d'Ascq, France \\ ${ }^{*}$ Corresponding author: karim.houni@telecom-lille1.eu
}

Received 31 July 2007; revised 6 January 2008; accepted 13 January 2008; posted 23 January 2008 (Doc. ID 85845); published 3 March 2008

\begin{abstract}
In the convergence context of identification technology and information-data transmission, the barcode found its place as the simplest and the most pervasive solution for new uses, especially within mobile commerce, bringing youth to this long-lived technology. From a communication theory point of view, a barcode is a singular coding based on a graphical representation of the information to be transmitted. We present an information theoretic approach for 1D image-based barcode reading analysis. With a barcode facing the camera, distortions and acquisition are modeled as a communication channel. The performance of the system is evaluated by means of the average mutual information quantity. On the basis of this theoretical criterion for a reliable transmission, we introduce two new measures: the theoretical depth of field and the theoretical resolution. Simulations illustrate the gain of this approach. (C) 2008 Optical Society of America
\end{abstract}

OCIS codes: $100.2960,110.3055$.

\section{Introduction}

Since the beginning of the 1970s, barcodes have been used for automatic identification and traceability of consumer goods and parcel post. Because of its simplicity, this technology quickly became inescapable in electronic data interchange. Nowadays this tendency has been strengthened with the development of tag reading and new services such as mobile ticketing.

Basically, a barcode conveys binary information to be decoded by a reading device. Barcode reading technologies are thus singular communication systems in which messages are graphically modulated before transmission. In several commercial applications $2 \mathrm{D}$ barcodes have progressively become widespread. However, 1D barcodes still flood the market of automatic identification, as they may require only a simple linear imager as a reader and are more robust to relative misplacement. The scientific litera-

0003-6935/08/081025-12\$15.00/0

(C) 2008 Optical Society of America ture has dedicated few works to $1 \mathrm{D}$ and $2 \mathrm{D}$ barcode coding. Pavlidis et al. [1,2] published a study related to information theory fundamentals outlining the process for barcode design using error detecting and correcting techniques. Tsi et al. [3] developed a method that allows the calculation of the working range in case of a CCD-based reader. Illumination level, image contrast, and the number of pixels per module - i.e., pixels per image bar width - have been presented to be three important limiting factors for the working range. From a technical point of view, additional parameters specify the performance of such readers, and we mention as instances resolution (smallest readable bar width), scanning angle, and acceptable skew or pitch rotations. Neither in the scientific literature nor in technical booklets are specifications given concerning the quantity of information that can be transmitted accurately, which is still the goal of the system.

Our first objective is to evaluate the average information quantity that can reliably be recovered by a digital imager reading a barcode. A barcode indeed 
contains an amount of information measured in bits and graphically mapped. As we are dealing with data transmission, we consider the problem from a communication point of view and model it according to the Shannon paradigm with a source of information, a channel, and the user of the information. The bar width, the camera's optical and electronic settings, and the relative positioning of the barcode and the sensor constitute the principal characteristics of the channel. We then address the problem of the information quantity that this channel can transmit accurately for a given probability distribution of its input alphabet. From this point of view, the average mutual information (AMI) between the input and the output of the channel is the commonly accepted measure in bits/barcode or bits/sequence of the amount of information transmitted through the channel.

The interest in information theory for optical system design and analysis is indeed a growing trend. Without being exhaustive, let us cite the works of O'Sullivan et al., who proposed a survey [4] on the image formation problem; in addition Brady and Neifeld published an introductive paper on information theoretic analysis of optical components [5]. More specifically, Wagner and Häusler in [6] and Huck et al. in [7] proposed information theoretic techniques for the optimization of image sensors to reduce redundancy in the acquisition process.

In a second phase of this work we evaluate some performance measures regarding the computed AMI. What are the minimum values required for factors such as bar width, positioning, noise, imprecise handling, or relative positioning of the reader and the barcode, in order to totally recover a given amount of information? From these limits we propose theoretical values for depth of field and resolution.

AMI is not a measure of these performances but a theoretical criterion needed to evaluate them. These performances are theoretical in the sense that they could be achieved under an infinitely hard hypothesis. Other criterion could be used to quantify them, such as visual acuity or digital imaging requirements. In [3] three factors are judiciously presented to evaluate the working range and resolution. All these factors have a minimum required value regarding complexity of the signal processing circuitry and decoding ability. The criteria in that case are pragmatic and related to technology advances and detection algorithm sophistication, whereas the AMI criterion is theoretical and disregards complexity and decoding delays. Moreover, among all the criteria noted above, only AMI is based on information, which is the object and goal of the system.

In digital transmission, error probability is a quality measure evaluated under optimal detection criteria and for a given barcode design. Like AMI, it is based on information, but in terms of acceptable loss of information bits in the transmissiondetection process. Computation of the error probability requires knowledge of the coding design and is intractable for channels with memory, so it is usually represented by its upper and lower bounds. AMI in return does not consider how the mapping of information to a graphical representation is realized. For these reasons we prefer AMI to the error probability criterion, as it is not limited to a particular code design and opens up the way to new code design motivated by the challenge of filling in the gap between the actually achieved performances and the theoretically possible ones.

The outline of this paper is as follows: in Section 2 distortions, including blur coming from both the free positioning of the barcode and the optical characteristics of the reader, are detailed. Section 3 is devoted to AMI. A stochastic model of the captured linear image is first developed. This model takes into account all the characteristics described earlier in Section 2. Then an algorithm based on the Monte Carlo method and the algorithm of [15] (the BCJR algorithm) proposed for the estimation of AMI. Section 4 shows the relevance of AMI, theoretical depth of field, and theoretical resolution through realistic examples of linear imagers. Influences of both the optical block characteristics and the free positioning of the barcode are also studied. Section $\underline{5}$ concludes this paper.

\section{System Description and Distortions}

The barcode is a graphical mapping of $n$ independent binary symbols into $n$ black or white $\omega$ width bars displayed on a white plane denoted $\Pi$. By convention, black and white bars, respectively, carry the symbols 0 and 1 with probability 0.5 . The right and left sides of the white background are considered start and end bars. They are indexed as 0 and $n+1$ [Fig. 1(a)]. The barcode is randomly oriented and located in front of a camera characterized by its focal length $f$, an aperture with specific shape (commonly circular or square), and a sensor located on a plane $\Pi^{\prime}$. We deal in this paper with a linear imager as the sensor with a row of $N$ square pixels of side length $\rho$. In the image plane each bar is represented by $N_{m}$ pixels. The Euclidean 3D space-denoted $e$-to which the barcode and the camera belong is specified by the Cartesian coordinate system $R(O, \vec{x}, \vec{y}, \vec{z})$. $O$ and $\vec{z}$ are, respectively, the optical center and the optical axis of the linear imager. The sensor plane $\Pi^{\prime}$ is orthogonal to the optical axis and is centered on $O^{\prime}=\left(0,0, Z_{c}\right)$. The barcode is arbitrarily moved, giving it six degrees of freedom, three translations and three rotations $(\psi, \phi, \theta)$ (Fig. 2). To each position corresponds an image projected onto $\Pi^{\prime}$, divided into $n+2$ areas $B C_{k}^{\prime}, k \in\{0, \ldots, n+1\}$ [Fig. 1(b)]. Finally, we assume that the linear imager reads the entire width of the barcode.

\section{A. Image Formation and Distortions}

We consider here the image formation process, highlighting the link between positioning and distortions. Camera characteristics and free positioning of the 


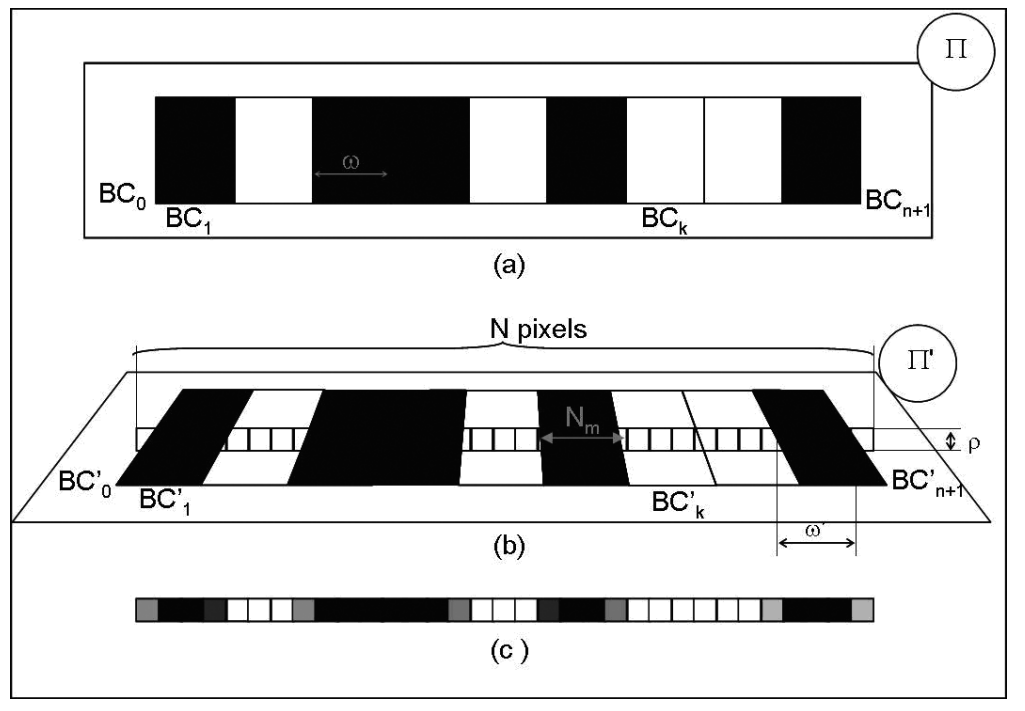

Fig. 1. Barcode displayed on (a) $\Pi$, (b) barcode projected on $\Pi^{\prime}$, and (c) barcode capture.

barcode induce four principal artifacts in the image formation process:

- Geometrical distortions,

- Radiant flux decay,

- Blur,

- Spatial nonalignment between projected bar frontiers and pixel borders.

\section{Geometrical Distortions}

Perspective projection on $\Pi^{\prime}$ induces geometrical distortions in the image of a barcode. The distortion is defined by the mathematical operator $T(\cdot)$ such that to any point $M(u, v, Z)$ belonging to $\Pi$ corresponds its projected point $M^{\prime}\left(u, v, Z_{c}\right)$ on $\Pi^{\prime}$ :

$$
M^{\prime}\left(T\left(u, v, Z_{c}\right)\right)=T(M(x, y, z)) .
$$

The positions of the bars of a barcode projected onto $\Pi^{\prime}$ are thus exactly known.

\section{Radiant Flux Decay}

We refer now to radiometric quantities to measure power or its distribution radiating to the sensor through the optical aperture $[8,9] . B C_{i}$ is an area reflecting or emitting light, and the radiance $X(M)$ measures the power per unit area per unit solid angle ( $\mathrm{W} \mathrm{m}^{-2} \mathrm{sr}^{-1}$ ) leaving a point $M$ in $B C_{i}$. We consider a homogeneous distribution over the surface, that is, $X(M)=X_{i} \forall M \in B C_{i}$, and perfect diffuse surfaces (Lambertian surfaces), where the radiance

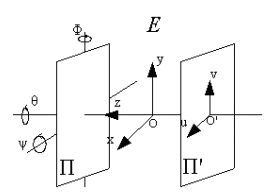

Fig. 2. Spatial settings and conventions. value is independent of the direction of illumination. The radiated power per unit solid angle $\left(\mathrm{W} \mathrm{sr}^{-1}\right)$ emanating from any direction having an angle $\beta=\beta(M, \Psi, \phi)$ with the normal to an elementary area $d S$ around $M$ is $X_{i} d S \cos (\beta)$. The solid angle $\Gamma$ subtended by the optical aperture of area $S_{a}$ from $M$ is

$$
\Gamma=S_{a} \cos \left(\alpha_{M}\right) / r_{M}^{2}
$$

with $r_{M}^{2}=\|\overrightarrow{O M}\|^{2}$ and $\alpha_{M}$ the angle between $\overrightarrow{O M}$ and $O \vec{z}$. For barcode positions in which $r_{M}$ is much larger than the optical aperture, the elementary area $d S$ illuminates the thin lens with a total power equal to

$$
\Phi(M) \approx X_{i} d S \cos (\beta) \frac{S_{a} \cos \left(\alpha_{M}\right)}{r_{M}^{2}}
$$

This expression shows that radiated power decays with $1 / r_{M}^{2}$ and falls as the barcode deviates and/or is rotated relative to the image plane. For a lens transmission factor equal to 1 , i.e., with no power loss between the lens and the sensor plane $\Pi^{\prime}$, the sensor irradiance $\left(\mathrm{W} \mathrm{m}^{-2}\right)$ at point $M^{\prime}\left(u, v, Z_{c}\right)$ of $B C^{\prime}{ }_{i}$ produced by the point $M\left(x, y, Z_{o}\right)$ of $B C_{i}$ is approximately

$$
I\left(M^{\prime}\right) \approx X_{i} \cos (\beta) \frac{S_{a} \cos \alpha_{M}}{r_{M}^{2}} \frac{d S}{d S^{\prime}}
$$

where $d S^{\prime}$ is the image patch of $d S$ centered on $M^{\prime}$. Using perspective projection geometry, one can establish the relation between the two elementary surfaces as

$$
\frac{d S}{d S^{\prime}}=\frac{\cos \alpha_{M}}{\cos \beta}\left(\frac{Z_{o}}{Z_{c}}\right)^{2}
$$


where $Z_{o}$ is the coordinate of point $M$ on the $O \vec{z}$ axis with $r_{M}=Z_{o} /\left|\cos \alpha_{M}\right|$, which yields

$$
I\left(M^{\prime}\right)=X_{i} \cos ^{4}\left(\alpha_{M}\right)\left(S_{a} / Z_{c}^{2}\right) .
$$

Finally, let us remark that $I\left(M^{\prime}\right)$ can be written as

$$
I\left(M^{\prime}\right)=\sum_{j=0}^{n+1} X_{j} \cos ^{4}\left(\alpha_{M}\right) \frac{S_{a}}{Z_{c}^{2}} 1_{B C_{j}^{\prime}}\left(M^{\prime}\right)
$$

with $1_{B C_{j}^{\prime}}(M)=1$ for $M^{\prime} \in B C_{j}^{\prime}$, 0 elsewhere.

\section{Optical Blur}

The blur is derived from the laws of both geometrical optics and Fourier optics. The former establishes the relation of the well-known thin lens formula [3] (Fig. 3), expressed for an object point $M\left(x, y, Z_{\text {of }}\right)$ and its focused image point $M^{\prime}\left(u, v, Z_{c}\right)$ as

$$
\frac{1}{Z_{c}}=\frac{1}{f}-\frac{1}{Z_{o f}} \text {. }
$$

$\Pi^{\prime}$ has a single corresponding object plane $\Pi$ placed on $Z_{o f}$, and a misplacement of $\Pi$ leads to an unfocused image on $\Pi^{\prime}$. In addition, Fourier optics emphasizes diffraction, which also gives rise to blur [10].

Thus, the blurring process is modeled by a global optical transfer function $H_{B}\left(f_{u}, f v\right)$, with $f_{u}$ and $f_{v}$ denoting spatial frequencies, and its equivalent point spread function $\left(h_{B}\right)$ on the image plane $H_{B}$ is a function of optical aperture shape [10]:

$$
H_{B}\left(f_{u}, f_{v}\right)=f(\text { optical aperture }) .
$$

The resulting irradiance $I_{B}\left(M^{\prime}\right)$ at any point $M^{\prime}$ of $\Pi^{\prime}$ is then the $2 \mathrm{D}$ convolution of $h_{B}$ with $I\left(M^{\prime}\right)$ :

$$
I_{B}\left(M^{\prime}\right)=\left(I * * h_{B}\right)\left(M^{\prime}\right),
$$

where $* *$ denotes $2 \mathrm{D}$ convolution.

In classical photography the spread of a light ray is identified as a blur circle (circle of confusion) on the sensor plane. As long as the blur circle does not exceed a given diameter, the image will appear to be in focus to the human viewer. One can then define a maximum permissible blur circle to ensure this latter condition according to human eye acuity. Displacements around the in-focus position give rise to two possible values of $Z_{o}$ corresponding to the maxi-

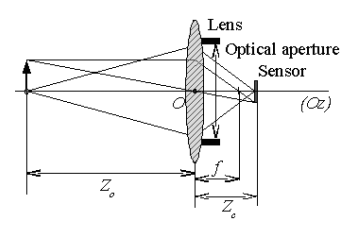

Fig. 3. Image formation diagram. mum permissible blur circle. One position is in front of the focus point $Z_{o f}$, and another one, which hereafter is denoted $Z_{d}$, beyond it. These two positions give rise to a measure well used by photographers, which is the depth-of-field region. As we deal with automatic processing of barcodes, we omit the eye resolution criterion and fix the maximum tolerable blur circle to one pixel size, with the resulting depth-of-field region as the reference.

\section{Spatial Nonalignment}

Each pixel integrates irradiance values in its area. Because of the random width value $\omega^{\prime}$ of the projected bar $B C^{\prime}{ }_{j}$, this operation may involve irradiance values resulting from one or more projected bars [Fig. 1(b)]. This creates interference between adjacent data, referred to as spatial nonalignment interference. Spatial nonalignment interference depends on $\omega^{\prime}$, on pixel size $\rho$, and on the shift amplitude between the edges of the projected image and the pixel. If the barcode is orthogonal to the $O \vec{z}$ axis, we can express $\omega^{\prime}$ simply as a function of the magnification factor and width $\omega$ of the bar. The number of pixels $N_{M}$ involved to generate the gray level $G_{i}$ can then be expressed as

$$
N_{m}=\left[\frac{Z_{c}}{Z_{o}} \frac{\omega}{\rho}\right]+k
$$

where [.] denotes the integer part of a real number and $k$ is a random variable taking its value from the set $\{1,2\}$. When this ratio decreases, the spatial nonalignment artifact becomes the preponderant cause of interference. $N_{m}$ refers to the number of pixels per module (the width of a projected bar).

The integration process over a pixel can be modeled as a moving average filter with an impulse response described as

$$
h_{I}(u, v, z)=\left(1 / \rho^{2}\right) 1_{[-\rho / 2, \rho / 2]^{2}}(u, v) \delta\left(z-Z_{c}\right) .
$$

The resulting image is then the convolution of $h_{I}$ with $I_{B}$ obtained in Eq. ( $\underline{7}$ ):

$$
I_{R}\left(M^{\prime}\right)=\left(I_{B} * * h_{I}\right)\left(M^{\prime}\right)=\left(I * * h_{B} * * h_{I}\right)\left(M^{\prime}\right) .
$$

$I_{R}\left(M^{\prime}\right)$ is then sampled at points $\left\{M_{k}^{\prime}, k \in\right.$ $\{1, \cdots, N\}\}$, denoted $\left\{J_{k}, k \in\{1, \cdots, N\}\right\}$, and corresponding to the centers of the $N$ pixels of the sensor and regularly spaced by distance $\rho$.

We aim now at studying the effects of all these distortions on the performances of an identification system, based on reading a barcode with a linear imager. To quantify these effects we adopt the AMI as a theoretical measure of achievable performance and as a novel criterion for optical characteristics and layout parameters. 


\section{Average Mutual Information}

\section{A. Definition}

Reading a barcode with a linear imager is an instance of a communication system. A sequence of gray levels $Y_{0: n+1}=\left(Y_{0}, Y_{2}, \ldots, Y_{n+1}\right)$ is captured as the received samples through a channel, and the barcode bars $X_{1: n}=\left(X_{1}, X_{2}, \ldots, X_{n}\right)$ are the transmitted symbols. In digital communication, AMI is considered a measure of the average information quantity that flows through a disturbed channel, having random sequences $X_{1: n}$ and $Y_{0: n+1}$ as its input and output, respectively. Let us first recall the general definition of AMI $[11,12]$.

Let $X_{1: n}=\left(X_{1}, \overline{X_{2}}, \ldots, X_{n}\right)$ and $Y_{0: n+1}=\left(Y_{0}, Y_{1}, \ldots\right.$, $\left.Y_{n+1}\right)$ be two sets of random variables. Each $X_{i} \quad(0 \leq$ $i \leq n+1)$ and each $Y_{i}(0 \leq i \leq n+1)$ takes its values from a finite alphabet $\Omega$ of symbols and from the set of real numbers $I R$. The AMI of a pair of random processes $I\left(X_{1: n} ; Y_{0: n+1}\right)$ measures the reduction in uncertainty in one of the two sequences when the second sequence is given. It can be expressed as

$$
I\left(X_{1: n} ; Y_{0: n+1}\right)=H\left(Y_{0: n+1}\right)-H\left(Y_{0: n+1} \mid X_{1: n}\right)
$$

with

$$
H\left(Y_{0: n+1}\right)=-\underset{Y}{E}\left[\log _{2}\left(p\left(Y_{0: n+1}\right)\right)\right]
$$

$$
\begin{aligned}
H\left(Y_{0: n+1} \mid X_{1: n}\right) & =-\underset{X, Y}{E}\left[\operatorname { l o g } _ { 2 } \left(p \left(Y_{0: n+1} \mid X_{1: n}=x_{1: n}, X_{0}\right.\right.\right. \\
& \left.\left.\left.=X_{n+1}=1\right)\right)\right] .
\end{aligned}
$$

Here $p\left(Y_{0: n+1}\right)$ is the probability density of $\left.Y_{0: n+1}\right)$, and $p\left(Y_{0: n+1} \mid X_{1: n}=x_{1: n} X_{0}=X_{n+1}=1\right)$ is the conditional probability density with reference to $x_{1: n}\left(X_{0}\right.$ and $X_{n+1}$ are always known to the receiver). In Eq. (12) expectation is processed over the random sequence $Y_{0: n+1}$, while in expression (13) it is done over the pair of random sequences $\left(X_{1: n}, Y_{0: n+1}\right)$.

$H\left(Y_{0: n+1}\right)$ defines the entropy of $Y_{0: n+1}$, which can be interpreted as the average uncertainty about this random sequence, and $H\left(Y_{0: n+1} / X_{1: n}\right)$ defines the conditioned entropy of $Y_{0: n+1}$ given $X_{1: n}$, interpreted as the part of uncertainty in $Y_{0: n+1}$ that can be resolved when $X_{1: n}$ is known.

Uncertainty and information can be regarded as two equivalent quantities. The information provided by a given realization will fill in the information gap, described as uncertainty. That is why in digital communication $I\left(X_{1: n} ; Y_{0: n+1}\right)$ measures the information flow through a channel [13].

The calculation of A $\overline{M I}$ requires modeling the channel on the basis of degradations described in Section 2. This modeling will allow us to calculate all the probabilistic functions needed to express entropies and thus $I\left(X_{1: n} ; Y_{0: n+1}\right)$.

\section{B. Channel Modeling}

In order to construct our model, we describe in the following the discrete image formation as a graylevel observation vector $\left(Y_{0} \cdots Y_{n+1}\right)^{T}\left[(\cdot)^{T}\right.$ being for transpose] obtained from input data vector $\left(X_{0} \cdots X_{n+1}\right)^{T}$. The sampled gray levels obtained at points $\left\{M^{\prime}{ }_{k}, k \in\{1, \cdots, N\}\right\}$ are denoted $\left\{J_{k}, k \in\right.$ $\{1, \cdots, N\}\}$. From Eqs. (4) and (10), we obtain

$$
J_{k}=\sum_{j=0}^{n+1} X_{j} h_{k, j}
$$

with

$$
h_{k, j}=C \frac{S_{a}}{Z_{c}^{2}}\left[\left(\cos \alpha_{M}\right)^{4} 1_{B C_{j}^{\prime}}\left(M^{\prime}\right) * *\left(h_{I} * * h_{B}\right)\right]_{M^{\prime}=M_{k}^{\prime}},
$$

where $C$ is a scale factor depending on the characteristics of the acquisition system (photosensitivity of the CCD sensor, integrating time, quantum efficiency, quantification transmittance function, etc.). We assume, in addition to the reasonable hypothesis, that variations of irradiance in the area of one pixel are negligible. Accordingly, the mean gray level $G_{i}$ of the captured bar $B C_{i}^{\prime}$ is the weighted summation

$$
G_{i}=\sum_{k=1}^{N} g_{i, k} J_{k},
$$

where $g_{i, k}$ is the normalized area of overlap between the $k$ th cell and $B C_{i}^{\prime}, 0 \leq i \leq n+1$. Equation (16) can also be written as

$$
G_{i}=\sum_{j=0}^{n+1} X_{j} \sum_{k=1}^{N} g_{i, k} h_{k, j} .
$$

In matrix notation, we have

$$
G^{(n+2)}=A X^{(n+2)},
$$

where $A$ is the so-called channel matrix and is an $(n+2) \times(n+2)$ matrix with elements $A_{i, j}=$ $\sum_{k=1}^{N} g_{i, k} h_{k, j}, \quad G^{(n+2)}$ and $X^{(n+2)}$ are the vectors $G^{(n+2)}=\left(G_{0} \ldots G_{n+1}\right)^{T}$, and $X^{(n+2)}=\left(X_{0} \ldots X_{n+1}\right)^{T}$.

An observed output channel $Y_{i}$ is associated with each transmitted symbol $X_{i} . Y_{i}$ results from the captured mean gray level, Eq. (18), corrupted by additive noise disturbances, modeled by a Gaussian whitenoise process:

$$
Y_{i}=\sum_{j=0}^{n+1} X_{j} A_{i, j}+B_{i} .
$$

Let us emphasize that, rewriting Eq. (19), it is straightforward to show that $Y_{0: n+1}$ is a hidden Markov model and that $F_{i}=\left(X_{i+L} \ldots X_{i} \ldots X_{i-L}\right)$ is a Markov process, $L$ representing the maximum memory length, i.e., $A_{i, j} \rightarrow 0$ for all $I$ and for $|j-i|>L$. 


\section{Average Mutual Information Computation}

AMI is obtained from computations of both the conditional entropy $H\left(Y_{0: n+1} \mid X_{1: n}\right)$ and the output entropy $H\left(Y_{0: n+1}\right) . H\left(Y_{0: n+1} \mid X_{1: n}\right)$ is fully determined by the entropy of the noise process $B_{0: n+1}[\underline{12}]$ :

$$
H\left(Y_{0: n+1} \mid X_{1: n}\right)=\frac{n+2}{2} \log _{2}\left(2 \pi e \sigma^{2}\right),
$$

where $\sigma^{2}$ is the power of $B_{i}$. The exact calculation of the output entropy $H\left(Y_{0: n+1}\right)(\underline{12})$ is, on the other hand, intractable. We then resort to the Monte Carlo method [14] to estimate it, which consists of an empirical estimation from an $N_{s}$ sample $\left\{p\left(y_{0: n+1}^{(s)}\right), s \in\right.$ $\left.\left\{1, \cdots, N_{s}\right\}\right\}$ :

$$
\hat{H}\left(Y_{0: n+1}\right)=-\frac{1}{N_{s}} \sum_{s=1}^{N_{s}} \log _{2}\left(p\left(y_{0: n+1}^{(s)}\right)\right),
$$

where each realization $y_{0: n+1}^{(s)}$ of $Y_{0: n+1}$ is obtained from Eq. (19) and where $X_{1: n}$ follows a uniform distribution on $\{0,1\}^{n}$ and each $B_{i}$ has a zero mean normal distribution with variance $\sigma^{2}$.

Using the Markovian property of $F_{-1: n+1}$ and because the original form of the BCJR algorithm $[15,16]$ is subject to numerical limitations, we resort here to a variant proposed in $[17,18]$. This variant is based, unlike classical BCJR, on the observation that $p\left(y_{k} \mid y_{0: k-1}\right)$ does not suffer underflow, so that one can estimate output entropy by

$$
\hat{H}\left(Y_{0: n+1}\right)=-\frac{1}{N_{s}} \sum_{s=1}^{N_{s}} \sum_{k=0}^{n+1} \log _{2}\left(p\left(y_{k}^{(s)} \mid y_{0: k-1}^{(s)}\right)\right),
$$

and the conditional probability density function $p\left(y_{k}^{(s)} \mid y_{0: k-1}^{(s)}\right)$ is given by the recursion of the two equations:

$$
\begin{aligned}
p\left(y_{k}^{(s)} \mid y_{0: k-1}^{(s)}\right)= & \sum_{f_{k-1}} \sum_{f_{k}} p\left(F_{k-1}=f_{k-1} \mid y_{0: k-1}^{(s)}\right) \\
& \times p\left(F_{k}=f_{k} \mid F_{k-1}=f_{k-1}\right) \\
& \times p\left(y_{k}^{(s)} \mid F_{k}=f_{k}, F_{k-1}=f_{k-1}\right)
\end{aligned}
$$

D. Average Mutual Information: Theoretical Criterion for Barcode Identification Performance

Our purpose is to assess AMI variations in the context of barcode reading with a $1 \mathrm{D}$ camera. As the barcode is freely positioned in front of the camera, variations of AMI depend on the overall layout, modeled in this work as a transmission channel by Eq. (19). In this equation the channel appears, being a memory channel with additive white Gaussian noise, where spatial interdependencies and radiant energy decay are summarized in matrix $A$. Blur and spatial nonalignment are responsible for the interdependency between neighboring pixels, while remote positions, rotations, and corresponding geometrical image reductions lead to received energy decays.

Let us consider a camera with optical and electronic (pixels and noise) settings and a barcode with an arbitrary position and a given bar width. AMI varies whenever one of these parameters varies. We aim first at studying how sensitive AMI is to changes in one of these parameters. We define the range in which one of the above parameters could vary such that AMI remains greater than a given value. Other criteria may be adopted, such as a maximal acceptable error probability or achievable decoding complexity. Both are practical and pragmatic. Error probability depends on barcode design, and decoding complexity depends on algorithmic sophistication and advances in technology. Both are related to a required signal-to-noise ratio (SNR) or to a minimalnoise-level power. The pertinence of AMI is that it is strictly related to the information quantity, which is specific for the system. In addition, it is a theoretical criterion that opens the way for new code design to approach theoretical limits. Performance based on the AMI criterion is independent of the state of the art in technology and could be considered an upper bound or a reference for actual and future work.

AMI is primarily a theoretical measure about the information quantity that can be transmitted accurately over a channel and for a given probability mass distribution at its input. In our context and for a given linear imager setting (including noise), AMI varies with $A$ as the barcode moves with respect

$$
p\left(F_{k}=f_{k} \mid y_{0: k}^{(s)}\right)=\frac{\sum_{f_{k-1}} P\left(F_{k-1}=f_{k-1} \mid y_{0: k}^{(s)}\right) P\left(F_{k}=f_{k} \mid F_{k-1}=f_{k-1}\right) p\left(y_{k}^{(s)} \mid F_{k}=f_{k}, F_{k-1}=f_{k-1}\right)}{\sum_{f_{k}} \sum_{k-1} P\left(F_{k-1}=f_{k-1} \mid y_{0: k}^{(s)}\right) P\left(F_{k}=f_{k} \mid F_{k-1}=f_{k-1}\right) p\left(y_{k}^{(s)} \mid F_{k}=f_{k}, F_{k-1}=f_{k-1}\right)} .
$$

At initialization, $y_{-1}^{(s)}$ is without information about $F_{-1}$ for any $s$, so that $F_{-1}$ is uniformly distributed: $P\left(F_{-1}=f_{-1} \mid y_{-1}^{(s)}\right)=1 / 2^{L}$. to the camera. One can then define an allowed region in space for which AMI remains superior to a fixed value. For pragmatic consideration let a barcode with 
given information content feed the channel. For example, let a barcode of 95 bars with on average 76 bits of information content feed the channel. To transmit all this information accurately, AMI must be at least equal to $76 \mathrm{bits} / \mathrm{sequence}$. All points in the space allowing this AMI will fill this range, which ensures efficient communication.

On the other hand, an important result from information theory states that noise limits the average information quantity that can be transmitted reliably through a channel. For a given matrix $A$, AMI decreases as noise power increases. The presence of noise then has an impact on the allowed region in space that ensures efficient communication.

Regarding the remarks in the preceding two paragraphs, we present AMI as an objective criterion for the estimation of what we shall define as the theoretical depth of field. In traditional photography, the geometrical computation of depth of field is determined by parameters such as the focal length, the aperture size, and a fixed permissible blur circle. Geometrical computations do not consider noise in expressing the depth of field, but only blur. We propose a theoretical depth-of-field measure related to AMI that varies according to channel matrix $A$ and/or noise power. As we shall show, this theoretical working region enlarges barcode reading capabilities beyond the usual optical performances inherited from classical photography. In all cases, it spurs research to conceive optimal barcode configurations and optimal detectors.

\section{Simulations and Results}

We evaluate and plot AMI variations for different positioning of the barcode in front of the camera and for different noise levels. These curves, giving AMI with respect to distance $Z_{o}$, encompass all the channel artifacts presented in Section 2 . We also plot AMI as a function of SNR in order to emphasize the influence of positioning on the channel memory and its effects on the transmissible information quantity.

In the following, we consider a linear imager with a square aperture of $2 d$ side length and focal length $f$. We define the $f$-number of the imager as the ratio $f / 2 d$. The linear sensor is fixed at distance $Z_{c}$ and is designed as a row of square pixels with side length equal to $5 \mu \mathrm{m}$. Because of the square aperture, the optical transfer function $H_{B}$ (Subsection 2.A.3) can be split into two functions, $H_{G}$ and $H_{D}$, related to geometrical and diffraction blur and defined by [10]

$$
\begin{aligned}
H_{G}\left(f_{X}, f_{Y}\right)= & \sin c\left(\frac{8 W_{m}\left(Z_{o}, \Delta Z\right)}{\nu}\left(\frac{f_{X}}{2 f_{0}}\right)\left(1-\frac{\left|f_{X}\right|}{2 f_{0}}\right)\right) \\
& \times \sin c\left(\frac{8 W_{m}\left(Z_{o}, \Delta Z\right)}{\nu}\left(\frac{f_{Y}}{2 f_{0}}\right)\left(1-\frac{\left|f_{Y}\right|}{2 f_{0}}\right)\right)
\end{aligned}
$$

where $\nu$ is the optical wavelength, $f_{0}=d / v Z_{c}$ is the optical cutoff frequency, $\Delta Z$ is the shift position with respect to the focused point set at $Z_{o}=Z_{o f}$, and
$W_{m}\left(Z_{o}, \Delta Z\right)$ is the shift function, such as

$$
\begin{gathered}
W_{m}\left(Z_{o}, \Delta Z\right)=\frac{d^{2}}{2}\left(\frac{1}{Z_{o}}-\frac{1}{Z_{o}+\Delta Z}\right) \\
H_{D}\left(f_{X}, f_{Y}\right)=\Lambda\left(\frac{f_{X}}{2 f_{0}}\right) \Lambda\left(\frac{f_{Y}}{2 f_{0}}\right)
\end{gathered}
$$

where $\Lambda(\cdot)$ is the triangle function.

The terms "long-range" (LR) or "short range" (SR) are used in the following to specify the optical configuration of the simulated imagers. More precisely, those cameras are

- A LR one with $Z_{\text {of }}=300 \mathrm{~mm}, f=7.6 \mathrm{~mm}$, $Z_{c}=7.8 \mathrm{~mm}$, and aperture $f / 8$;

- A compact LR (cLR) one with same $Z_{\text {of }}=$ $300 \mathrm{~mm}$ but $f=1.492 \mathrm{~mm}, Z_{c}=1.5 \mathrm{~mm}$, and aperture $f / 1.4$;

- A SR one with $Z_{\text {of }}=45 \mathrm{~mm}$ and aperture $f / 8$.

These specifications, quite arbitrary although referring to common industrial equipment, were chosen to study the effect of blur and spatial nonalignment separately.

We simulate a barcode pattern composed of 95 bars of constant width $\omega$ set to $1 \mathrm{~mm}$. For each bar $B C_{i}$ we consider a random symbol $X_{i}$ taking its values from a binary set $\Omega$ of arbitrary radiance quantities. Noise power spectral density $N_{o}$ is set to values that ensure a SNR varying from -10 to $35 \mathrm{~dB}$ with respect to radiance. Common values of an array sensor SNR are between 0 and $50 \mathrm{~dB}$ [19]. Our lower SNR range is set to determine whether, when performance is measured with AMI, efficient transmission remains achievable with noisier systems.

\section{A. Theoretical Depth of Field}

Let $Z_{o}$ be the distance between the center of the barcode and the thin lens on the $\vec{O} z$ axis (see Fig. 2). In this paragraph we consider cameras with only one possible focused distance, $Z_{o}=Z_{o f}$. Let $Z_{o}$ vary from $Z_{o f}$ to $2 Z_{o f}$ and the orientation of $B C$ vary from 0 to $0.2 \pi$ relative to the angle $\phi$. We first consider the LR linear imager. The geometrically computed farextreme point of the depth-of-field region is about $380 \mathrm{~mm}$. These computations are obtained for a permissible blur circle diameter equal to a pixel width. AMI variations are plotted with respect to the distance $Z_{o}$ [Fig. 4(a)] and angle $\phi$. The mean channel memory length $L$ for this imager varies from two to four symbols.

For a low-noise power quantity, AMI losses are less than $1 \%$ even in a region beyond the classically computed depth-of-field region. Conceptually speaking, all information can undeniably be recovered in a noiseless channel after an exact estimate of its matrix $A$, whatever $A$ is, in other words, however large the blur circle is. This result confirms the need to define a theoretical depth of field related to an accep- 


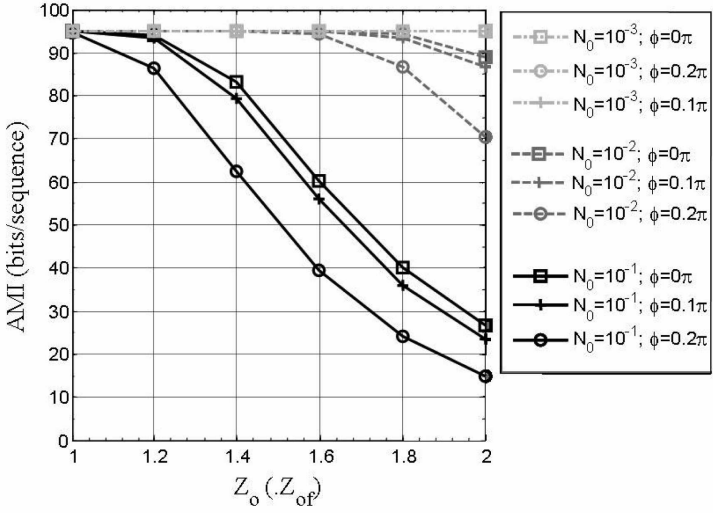

(a)

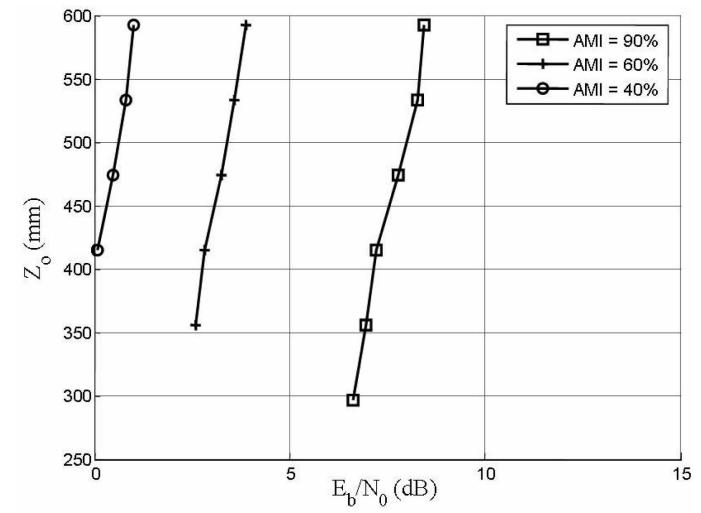

(b)

Fig. 4. (a) LR imager AMI variations regarding $Z_{0}$. (b) LR theoretical depth of field for fixed AMI levels.

table loss of AMI. Nevertheless, we note a drastic decrease of AMI in the case of a noisy channel. As noise is a limiting factor for AMI, it consequently limits the theoretical depth-of-field region. For instance, if we consider a minimum acceptable AMI of 90 bits/ sequence, then the far-extreme point of the theoretical depth of field goes beyond $2 Z_{\text {of }}$ at $N_{o}=10^{-3}$, reduces to $2 Z_{o f}$ at $N_{o}=10^{-2}$, and is confined to about $380 \mathrm{~mm}$ at $N_{o}=10^{-1}$. For positions in front of $Z_{o f}$ AMI losses are less than $1 \%$ for all simulated noise power quantities; so the theoretical depth of field is equal to the far-extreme point measured on the curve. In Table 1 we note some theoretical depthof-field distances measured for different acceptable AMI values for $N_{o}=10^{-1}$. We sketch in Fig. $4(\mathrm{~b})$ variations of the theoretical depth of field as a function of the SNR $E_{b} / N_{0}, E_{b}$ being the mean received binary energy. Figure $4(\mathrm{~b})$ gives a global view of theoretical depth-of-field notion: it depicts the admitted barcode position range for a fixed AMI level. $E_{b}$ is a function of the integrated irradiance on the image plane; that is, it encompasses the effects of illumination variation, spatial nonalignment, geometrical distortions, and blur.

In the following we study the influence of optical characteristic changes on the theoretical depth of field. Classical photography rightly states that, with the same aperture size, reducing the focal length leads to a greater depth of field and, inversely, this depth of field is reduced for focusing at a shorter distance.

We consider for this purpose the cLR linear imager. As mentioned above, a shorter focal length gives rise to a larger depth-of-field region. The far-extreme point of the computed standard depth of field is indeed $4.2 \mathrm{~m}$. But for the theoretical depth of field we observe a reverse phenomenon. The mean channel memory length varies from two to six symbols, and for an acceptable AMI of $90 \mathrm{bits} / \mathrm{sequence}$, the far-extreme point of the theoretical depth of field approaches $2 Z_{o f}$ at $N_{0}=10^{-3}$, is reduced to $400 \mathrm{~mm}$ at $N_{0}=10^{-2}$, and is in front of $Z_{\text {of }}$ at $N_{0}=$ $10^{-1}$ [Fig. 5(a)].

Values corresponding to other AMI limits are noted in Table 1 for $N_{0}=10^{-1}$, and Fig. 5(b) shows how these distances vary as a function of $E_{b} / N_{0}$. One can clearly see how this parameter is reduced compared with the earlier case. This reduction is especially due to other terms of intersymbol interference, which are not taken into account when computing the depth of field geometrically. We will see below that spatial nonalignment is the principal cause of this reduction.

We now consider the optical block focusing on a closer point object $Z_{o f}=45 \mathrm{~mm}$, introduced above as a SR linear imager. The far-extreme point of the standard depth of field $Z_{d}$ is about $50 \mathrm{~mm}$. Simulation results for AMI variations are presented in Fig. 6(a).

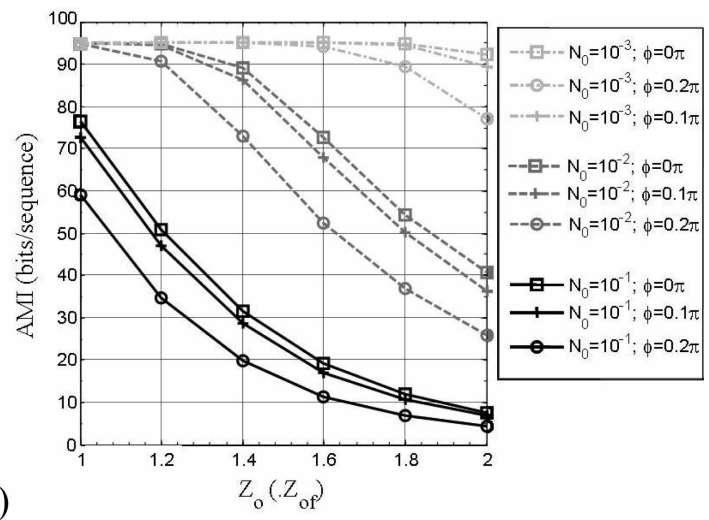

(a)

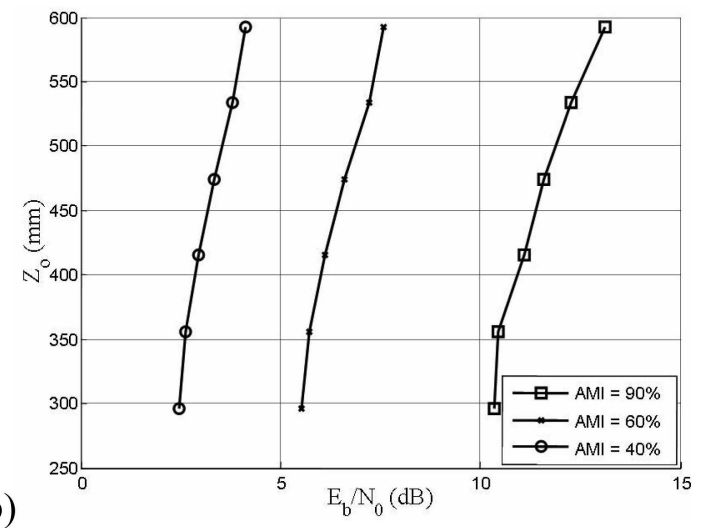

Fig. 5. (a) cLR imager AMI variations regarding $Z_{0}$. (b) $c L R$ theoretical depth of field for fixed AMI levels. 
Table 1. Barcode Positions under AMI Constraint when Facing the Camera $\left(N_{0}=10^{-1}\right)$

\begin{tabular}{|c|c|c|c|}
\hline AMI (bits/sequence) & $\begin{array}{c}Z_{o}(\mathrm{~mm}), \mathrm{LR} \\
f=7.6 \mathrm{~mm} ; Z_{o f}=300 \mathrm{~mm}\end{array}$ & $\begin{array}{c}Z_{o}(\mathrm{~mm}), \mathrm{cLR} \\
f=1.49 \mathrm{~mm} ; Z_{o f}=300 \mathrm{~mm}\end{array}$ & $\begin{array}{c}Z_{o}(\mathrm{~mm}), \mathrm{SR} \\
f=7.6 \mathrm{~mm} ; Z_{o f}=45 \mathrm{~mm}\end{array}$ \\
\hline 90 & 380 & 280 & 80 \\
\hline 60 & 480 & 340 & 100 \\
\hline 40 & 540 & 380 & 125 \\
\hline
\end{tabular}

Whereas simulations show that $L$ remains a twosymbol-long memory, theoretical depth of field values for different acceptable AMI quantities at $N_{0}=10^{-1}$ are in Table 1 . Figure 6(b) represents variations of this theoretical limit with respect to of $E_{b} / N_{0}$. Recalling classical photography statements, the ratio $Z_{d} / Z_{\text {of }}$ diminishes for focusing on closer objects. A quick comparison with the LR linear imager shows that this statement does not concord with the results obtained with the theoretical depth of field. This is because the LR linear imager is limited by other terms of intersymbol interference, whereas the SR camera is not. In the subsequent paragraphs we analyze the implications of the use of an autofocus and how the effects of other sources of distortions affect the AMI and therefore the theoretical depth of field.

\section{B. Autofocusing Effect on Average Mutual Information}

Diffraction, unfocusing, and spatial nonalignment produce intersymbol interference that leads to AMI

(a)
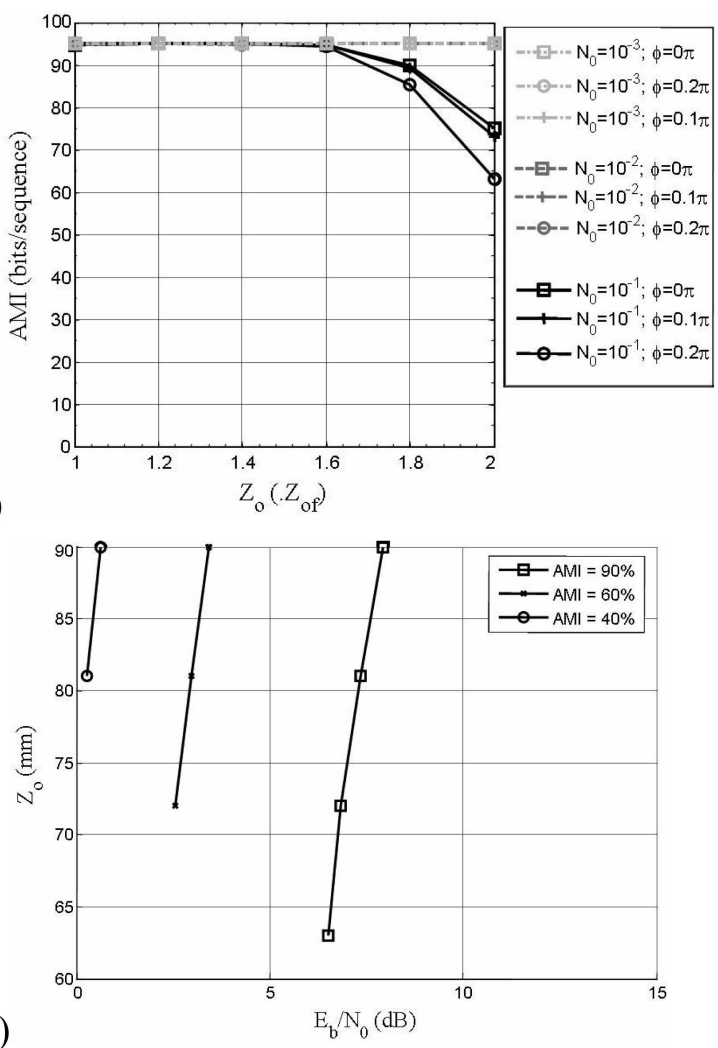

Fig. 6. (a) SR imager AMI variations in $Z_{0}$. (b) SR theoretical depth of field for fixed AMI levels. degradations. Below we measure the effect of autofocusing on the system.

Starting with the LR imager, we plot AMI variations by varying the center of the barcode location $Z_{o}$, considered here the focused point. These variations are reported in Figs. 7(a) and 7(b) with respect to $Z_{o}$ and the SNR $E_{b} / N_{0}$, respectively. Figure 7(b) shows different curves, each one corresponding to a different position of the barcode, and so to a different channel matrix $A$. When the barcode is parallel to the sensor plane the light energy spread due to aberration is null [i.e., $H_{G}\left(f_{x}, f_{y}\right)=1$ in Eq. (25)], as $\Delta Z=$ 0 for all points in the object plane and $\overline{W_{m}}\left(Z_{o}, \Delta Z\right)=$ 0 . Only the diffraction term is present in the global

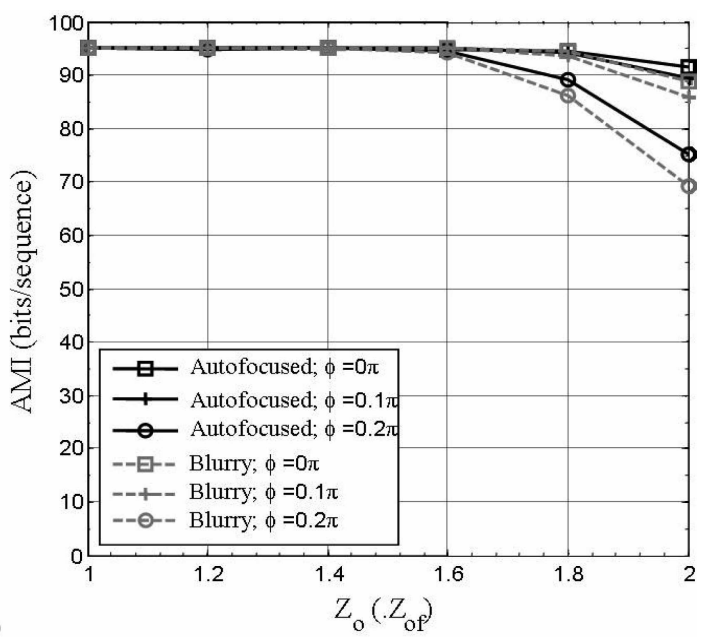

(a)

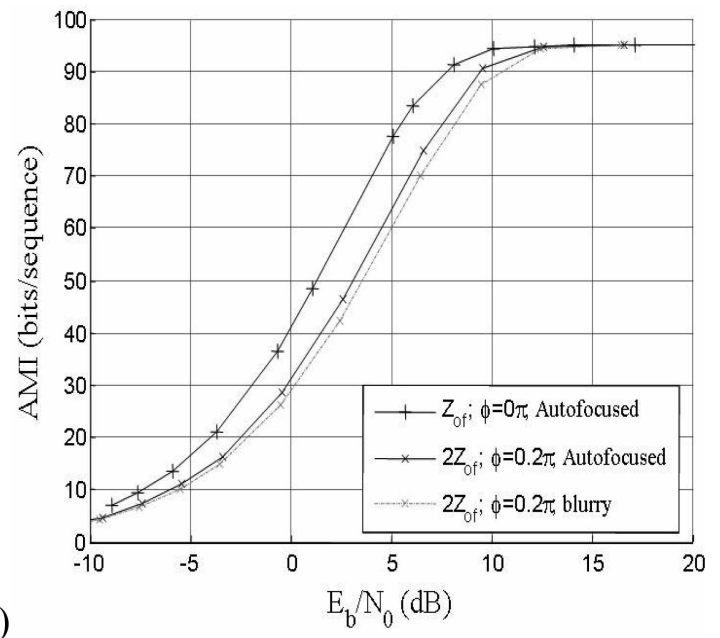

(b)

Fig. 7. LR AMI variations in the case of normal transmission and autofocus with respect to (a) $Z_{0}$ (with $N_{0}=10^{-1}$ ), (b) SNR. 
transfer function. Figures 7 show that autofocus slightly enhances (a few bits/sequence) the overall performances of the LR camera configuration. We will see below that for the SR camera autofocus gives substantial ameliorations.

From imprecise handling, the object may be rotated by angle $\phi$ with respect to the $O \vec{y}$ axis. In this case, the shift function $W_{m}\left(\boldsymbol{Z}_{o}, \Delta \boldsymbol{Z}\right)$ is not null for all points in the object plane such as $\Delta Z \neq 0$, and the aberration term remains in the transfer function. In Fig. 7(b) no significant effects on AMI could be measured for $\phi=0.2 \pi$.

We now emphasize AMI losses caused when spatial nonalignment is the preponderant factor in the intersymbol interference channel. We encounter this situation when the image bar width is almost equal to the pixel side length, which is the case for a cLR imager. AMI variations after autofocusing are plotted in Fig. 8 .

Compared with the LR camera, where $Z_{c}=$ $7.8 \mathrm{~mm}$, the sensor plane position $Z_{c}=1.5 \mathrm{~mm}$ of the cLR camera is approximately one fifth. These two cameras have the same nominal focused point $Z_{o f}=300 \mathrm{~mm}$; so the magnification factor $\left(Z_{c} / Z_{o f}\right)$

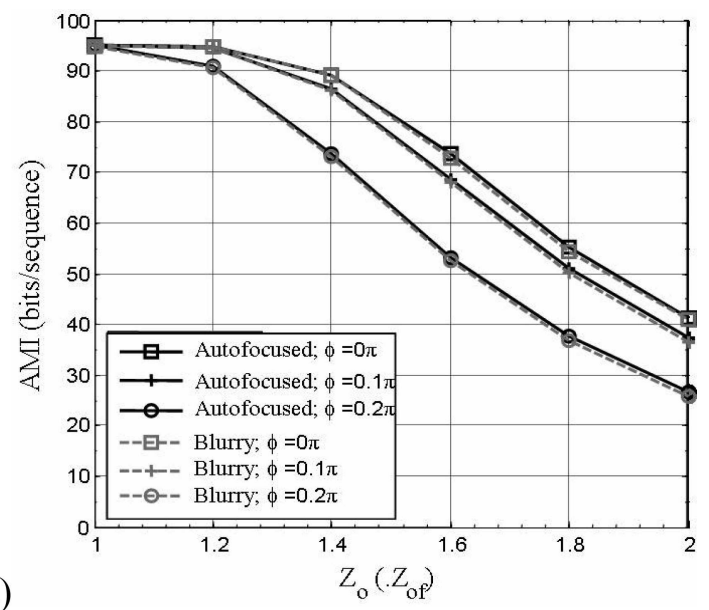

(a)

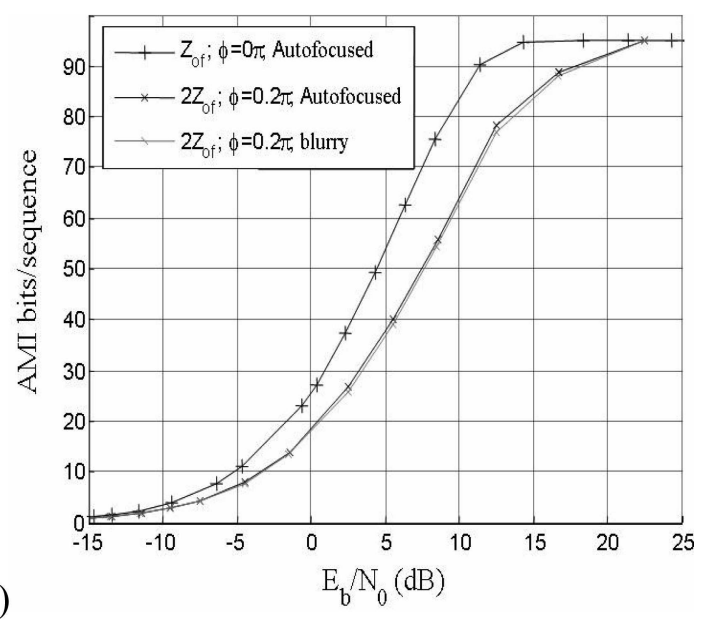

Fig. 8. cLR AMI variations in the case of normal transmission and autofocus with respect to (a) $Z_{0}$ (with $N_{0}=10^{-2}$ ), (b) SNR. is approximately five times smaller for the cLR camera. This leads to a smaller projected image $B C^{\prime}$. The bars are therefore captured by fewer pixels, increasing the effects of intersymbol interference and thus rendering the communication system more sensitive to noise. The number of pixels per module $N_{m}$ [Eq. (8)] varies from 2 to 1 when moving from $Z_{o f}$ to $2 Z_{\text {of }}$. Figure $\underline{8}$ plots AMI after autofocusing, and it is obvious that focus handling brings no relevant improvement in AMI. Consequently, spatial nonalignment appears to be the principal cause of intersymbol interference. Compared with Fig. 7 of the LR settings, Fig. 8 shows how degradations due to the smaller projected image are significant. One can then classify this cLR camera as a detector-limited linear imager from a technological point of view, and it is rather limited to large-size barcode design.

Finally, an autofocus process is also added to the SR camera. The results are presented in Fig. 9, where AMI is much more improved than for $L \bar{R}$ and cLR. Indeed, autofocus brings a gain of up to $20 \mathrm{bits} / \mathrm{sequence.} \mathrm{In} \mathrm{fact,} \mathrm{with} \mathrm{SR} \mathrm{camera} \mathrm{settings,}$ spatial nonalignment has a low impact: $N_{m} \geq 20$ within the range $\left[Z_{o f}: 2 Z_{o f}\right]$. This is supported by

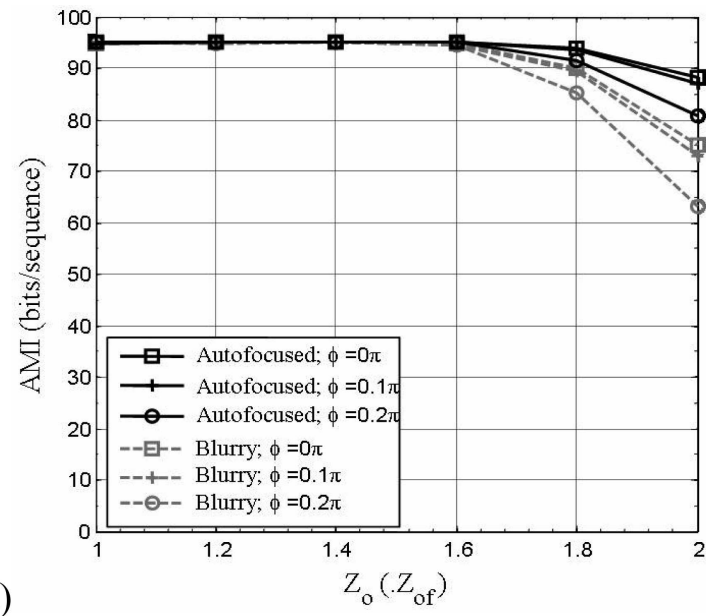

(a)

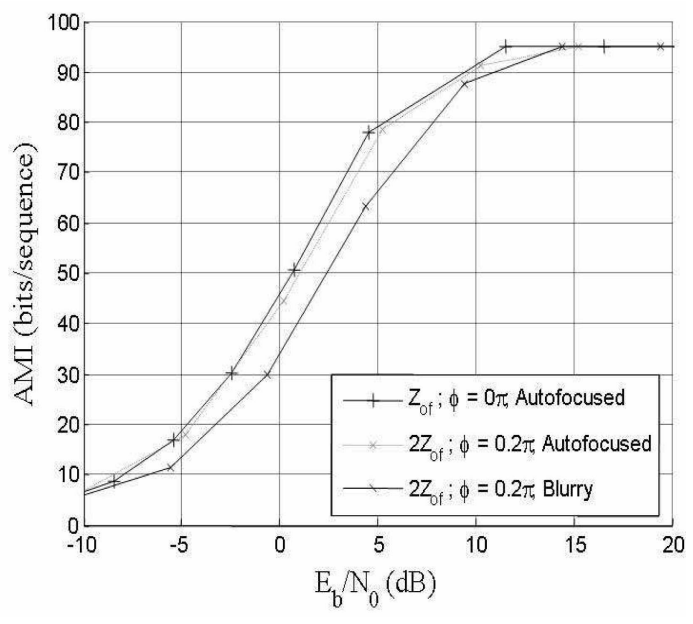

(b)

Fig. 9. SR AMI variations in the case of normal transmission and autofocus with respect to (a) $Z_{0}$ (with $N_{0}=10^{-1}$ ), (b) SNR 
(a)

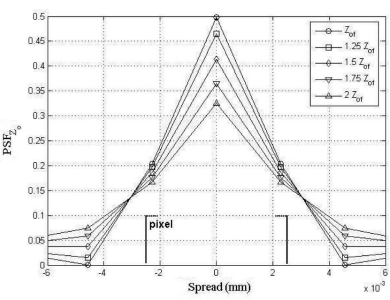

(b)

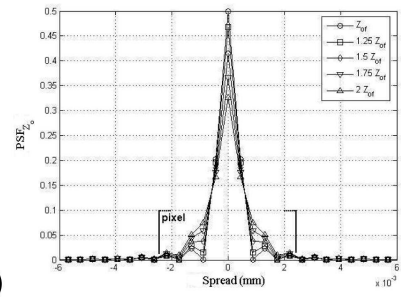

Fig. 10. Point spread function variations for different $Z_{0}$ for (a) LR, (b) cLR, and (c) SR optics.

Fig. 9(b), where AMI curves plotted with respect to the SNR for all focused positions are approximately the same as the curve of the nominal position $Z_{o f}$. This camera is very sensitive to displacements, but once autofocusing is used, the large magnification factor yields a substantial tolerance to free handling.

Last, plotting the variations of the fixed focal length version PSFs of the three optical blocks for different $Z_{o}$ highlights their very different behavior relative to displacement. The curves are plotted in Fig. 10.

\section{Theoretical Resolution}

We now relate AMI to resolution, which we define as the minimal bar width needed to recover a specific amount of information. We thus introduce a theoretical value for this resolution after inspection of curves relating AMI to the SNR. Considering, for instance, an AMI curve at a distance $2 Z_{o f}$, one can move along its trajectory for different SNR values, which could be obtained by fixing $N_{0}$ and varying the radiated energy. This latter may vary with bar width $\omega$, and this enables us to draw a direct link between AMI and $\omega$.

As an illustration, we consider the LR and cLR imagers (Fig. 11). To ensure a reliable transmission of 80 bits over $\overline{94}$ transmitted bars at $N_{0}=10^{-2}$, the minimal required bar width is $0.25 \mathrm{~mm}$ at $Z_{o}=2 Z_{\text {of }}$ for the LR settings and shifts to $0.5 \mathrm{~mm}$ and $1.7 \mathrm{~mm}$, respectively, at the same positions for the cLR camera. The main reason for these stronger requirements for the cLR is the smaller magnification factor within the range of measurements. What is in fact important for AMI performance is to ensure a good signal-to-interference ratio by augmenting the magnification factor, providing a system more compatible with higher-density codes. Thus the LR camera has a higher resolution despite the fact that it is less robust to displacements, as classical optics suggests.
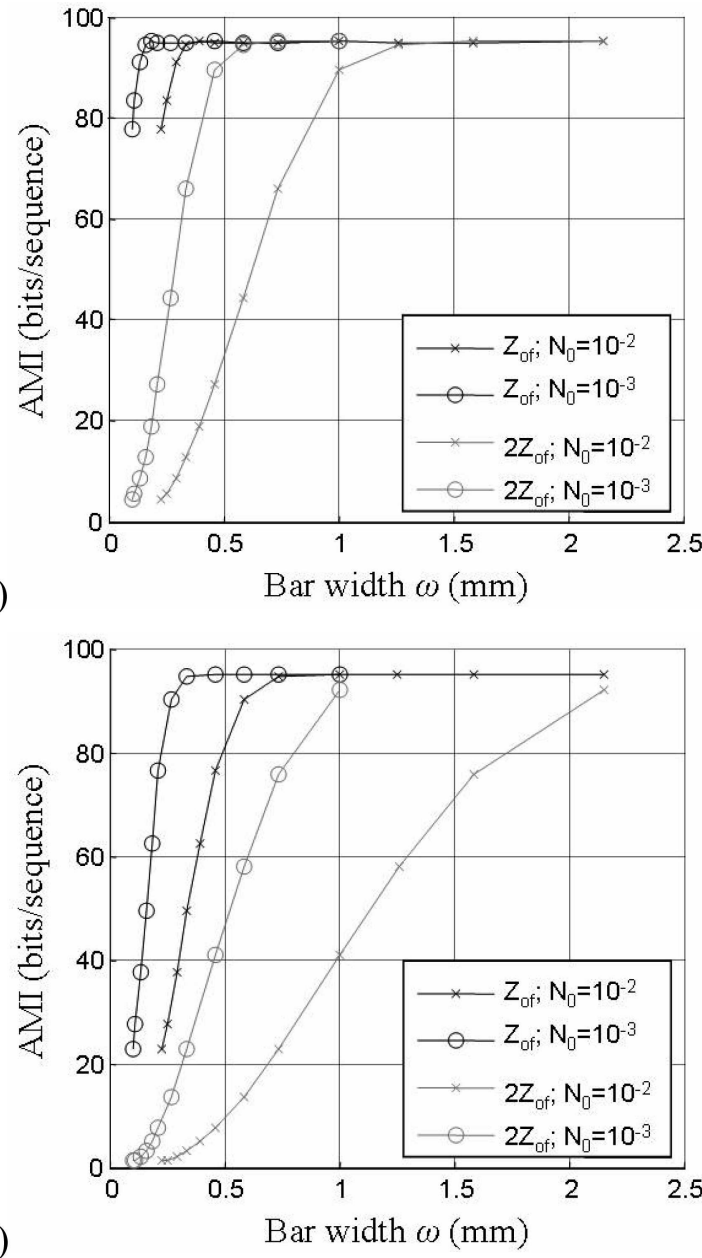

(b)

(c)

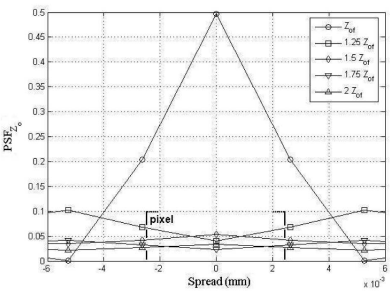

Fig. 11. AMI constrained minimal bar width estimations for (a) LR and (b) cLR imagers.

\section{Conclusion}

We have presented a novel method for performance analysis of $1 \mathrm{D}$ barcode reading with linear imagers. We have demonstrated that standard optics does not yield a measure of the transferable information, especially because it does not take into account noise and spatial nonalignment of the barcode. As a consequence, we have considered barcode displaying capturing - decoding as a digital communication system.

The depth of field and the resolution have been redefined according to the objective mathematical measure of the transferable information known as average mutual information (AMI). Through the 
model we have developed, AMI encompassed all the channel artifact sources (blur, geometrical distortions, and noise) so that its estimation draws a direct link between system design and information theory.

The performance of common industrial barcode linear imagers has been analyzed in terms of AMI, theoretical depth of field, and resolution. The AMIbased analysis has also been compared with the standard optical one, and its relevance has been highlighted for the design of a communication system based on 1D barcode technology.

\section{References}

1. T. Pavlidis, J. Swartz, and Y. P. Wang, "Fundamentals of bar code information theory," Computer 23(4), 74-86 (1990).

2. T. Pavlidis, J. Swartz, and Y. P. Wang, "Information encoding with two-dimensional bar codes," Computer 25(6), 18-28 (1992).

3. D. Tsi, E. Marom, J. Katz, and J. Swartz, "System analysis of CCD-based bar code readers," Appl. Opt. 32, 3504-3512 (1993).

4. J. A. O'Sullivan, R. E. Blahut, and D. L. Snyder, "Informationtheoretic image formation," IEEE Trans. Inf. Theory 44, 2094-2123 (1998).

5. D. Brady and M. A. Neifeld, "Information theory in optoelectronic systems: introduction to the feature," Appl. Opt. 39, 1679-1680 (2000).

6. C. Wagner and G. Häusler, "Information theoretical optimization for optical range sensors," Appl. Opt. 42, 5418-5426 (2003)
7. F. O. Huck, C. L. Fales, R. Alter-Gartenberg, S. K. Park, and Z. Rahman, "Information-theoretic assessment of sampled imaging systems," Opt. Eng. 38, 742-762 (1999).

8. R. E. Jacobson, G. G. Attridge, N. R. Axford, P. Ward, and S. F. Ray, Manual of Photography-Photographic and Digital Imaging, 9th ed. (Focal, 2000).

9. D. A. Forsyth and J. Ponce, Computer Vision (Pearson Education, 2003).

10. J. W. Goodman, Introduction to Fourier Optics, 2nd ed. (McGraw Hill, 1996).

11. R. G. Gallager, Information Theory and Reliable Communication (Wiley, (1968).

12. T. J. Cover, Elements of Information Theory (Wiley, 1991).

13. S. Benedetto, E. Biglieri, and V. Castellani, Digital Transmission Theory (Prentice Hall, 1987).

14. M. Mascagni, Lecture notes from Advanced Monte Carlo Methods I \& II: An ETHZ/SAM Course (Florida State University and Swiss Federal Institue of Technology, 2005-2006).

15. L. R. Bahl, J. Cocke, F. Jelinek, and J. Raviv, "Optimal decoding of linear codes, for minimizing symbol error rate," IEEE Trans. Inf. Theory 20, 284-287 (1974).

16. L. E. Baum, T. Petrie, G. Soules, and N. Weiss, "A maximization technique occurring in the statistical analysis of probabilistic functions of Markov chains," Ann. Math. Stat. 41, 164171 (1970).

17. C. Berrou and A. Glavieux, "Near optimum error correcting coding and decoding : turbo codes," IEEE Trans. Commun. 44, (1996).

18. D. Arnold and H.-A. Loeliger, "On the information rate of binary-input channels with memory," in IEEE International Conference on Communications, 2001. ICC 2001 (IEEE, 2001), pp. 2692-2695.

19. G. C. Holst, CCD Arrays, Cameras and Displays, 2nd ed. (SPIE , 1998). 